\title{
Comparación de la tasa de cesáreas de dos maternidades públicas del Uruguay
}

\author{
Comparison of the caesarean section rate of two publics health maternity in \\ Uruguay
}

\section{Comparação da taxa de cesariana de duas maternidades públicas no Uruguai}

\author{
Florencia Feldman'1, Gina Del Villar², PamelaGrimaldi³, Santiago Scasso4, Grazzia Rey ${ }^{5}$
}

\section{RESUMEN}

Se realizó un analisis de la tasa de cesáreas en dos maternidades públicas de referencia de Uruguay (Hospital de Clinicas y Centro Hospitalario Pereira Rossell) utilizando la clasificación de Robson para compararlas entre sí, mediante un estudio observacional, descriptivo, retrospectivo y transversal en un periodo de 10 años y 10 meses (2009-2019).

Se analizaron 85.526 nacimientos $(7.685(8,9 \%)$ en el Clínicas vs 77.841 (91.1\%) Pereira Rossell). El porcentaje de cesáreas por año en el Clínicas fue $49,2 \% \pm 5$ vs $29,3 \% \pm 3$ en Pereira Rossell. Los grupos de Robson más prevalentes fueron 1,5A y 10 en el Clinicas vs 3, 1 y $5 \mathrm{~A}$ en Pereira Rossell. En ambos centros los grupos con mayor contribución relativa a la tasa global de cesáreas fueron: $5 \mathrm{~A}, 10$ y 1. Ambos centros presentan un aumento en la tasa de cesárea en la última década, pese a que se asisten poblaciones dispares entre cada uno de ellos. Se debe seguir buscando estrategias que ayuden a reducir la tasa de cesáreas principalmente en pacientes sin cesáreas anteriores o con una única cesárea previa, en caso de no presentan contraindicaciones para el parto vaginal.

Palabras clave: cesárea, prevalencia, maternidades, clasificación de Robson.

\section{ABSTRACT}

An analysis of the caesarean section rate was carried out in two reference public maternity wards in Uruguay (Hospital de Clinicas and Centro Hospitalario Pereira Rossell) using Robson's classification to compare them with each other, through an observational, descriptive, retrospective and cross-sectional study in a period 10 years and 10 months (2009-2019).

85.526 births were analyzed $(7,685(8.9 \%)$ in the Clinics vs 77,841 (91.1\%) Pereira Rossell). The percentage of caesarean sections per year in the Clinics was $49.2 \% \pm 5$ vs $29.3 \% \pm 3$ in Pereira Rossell. The most prevalent Robson groups were 1, $5 \mathrm{~A}$ and 10 in the Clinicas vs 3,1 and $5 \mathrm{~A}$ in Pereira Rossell. In both centers, the groups with the highest relative contribution to the overall rate of cesarean sections were: $5 \mathrm{~A}, 10$ and 1 . Both centers show an increase in the rate of cesarean section in the last decade, despite the fact that different populations are attended between each of them. Strategies should continue to be sought to help reduce the rate of cesarean sections, mainly in patients without previous cesarean sections or with a single previous cesarean section, if they do not present contraindications for vaginal delivery.

Keywords: caesarean section, prevalence, maternity, Robson classification

RESUMO
Foi realizada análise da taxa de cesárea em duas maternidades públicas de referência do Uruguai (Hospital de Clínicas e Centro Hospitalario Pereira Rossell), utilizando a classificação de Robson para compará-las, por meio de estudo observacional, descritivo, retrospectivo e transversal. em um período de 10 anos e 10 meses (2009-2019)

Foram analisados 85.526 partos (7.685 (8.9\%) nas Clínicas vs 77.841 (91,1\%) Pereira Rossell). A porcentagem de cesarianas por ano nas Clinicas foi de $49,2 \% \pm 5$ vs $29,3 \% \pm 3$ em Pereira Rossell. Os grupos de Robson mais prevalentes foram 1, 5A e 10 nas Clinicas vs 3, $1 \mathrm{e}$ $5 \mathrm{~A}$ em Pereira Rossell. Em ambos os centros, os grupos com maior contribuição relativa para a taxa global de cesárea foram: 5A, 10 e 1. Ambos os centros apresentam aumento da taxa de cesárea na última década, apesar de diferentes populações serem atendidas entre cada um deles. Estratégias devem continuar a ser buscadas para ajudar a reduzir a taxa de cesárea, principalmente em pacientes sem cesárea anterior ou com cesárea única, se não apresentarem contra-indicações para parto normal.

Palavras-chave: cesarea, prevalencia, maternidades, classificação de Robson.

\section{INTRODUCCIÓN}

A lo largo de las últimas décadas, ha habido un aumento progresivo de la tasa de cesáreas en la mayoria de los paises, pero no se conocen por completo las razones que han impulsado esta tendencia ${ }^{(1 .}$ 2). El aumento de la tasa de cesárea constituye un importante motivo de preocupación en el ámbito de la salud pública, se han visto incrementos de hasta un $70 \%$, lo cual provoca debates a nivel mundial debido a los posibles riesgos maternos y perinatales asociados con este aumento, la inequidad en el acceso y las cuestiones relativas al costo ${ }^{(3-5)}$. La Organización Mundial de la Salud (OMS) sostiene que no existen beneficios adicionales con proporciones de cesárea por encima del 15\%(3, 6).

En el 2014, la OMS efectuó una revisión sistemática de los estudios disponibles relacionados con propuestas y análisis de tasa ideal de cesárea en poblaciones o paises determinados. Asimismo, propuso utilizar el Sistema de Clasificación de Robson como estándar

1 Residente de la Clinica Ginecotocológica B. Facultad de Medicina, Universidad de la República. Uruguay. Correo electrónico: flofel_0236@hotmail.com Correspondencia: Pedro Francisco Berro1115 apto 601. Montevideo, Uruguay. 
global para evaluar y comparar las tasas de cesárea y darle seguimiento en los establecimientos sanitarios a lo largo del tiempo y compararlos entre ellos ${ }^{(2,3)}$. Ésta propone un método que discrimina la población atendida en diez grupos, representados en la Tabla 1. La misma tiene en cuenta cuatro conceptos obstétricos y permite identificar los grupos que más aportan a la cifra global de cesárea, hacer comparaciones en igualdad de condiciones entre instituciones y dentro de la misma institución en el tiempo, permitiendo planear intervenciones especificas ${ }^{(2,3,6)}$.

Tabla 1. Categorias de Robson y criterios de inclusión para cada grupo1

\begin{tabular}{|c|c|}
\hline $\begin{array}{l}\text { Categoria } \\
\text { de } \\
\text { Robson }\end{array}$ & Características del grupo \\
\hline 1 & $\begin{array}{l}\text { Nuliparas con un feto único en presentación cefálica, de } 37 \text { semanas o más de } \\
\text { embarazo, que han iniciado el parto de forma espontánea }\end{array}$ \\
\hline 2 & $\begin{array}{l}\text { Nuliparas con un feto único en presentación cefálica, de } 37 \text { semanas o más de } \\
\text { embarazo, que han sido sometidas a inducción o a cesárea antes del inicio del } \\
\text { parto }\end{array}$ \\
\hline 3 & $\begin{array}{l}\text { Multíparas sin cesárea previa, con un feto único en presentación cefálica, de } \\
37 \text { semanas o más de embarazo, que han iniciado el parto de forma } \\
\text { espontánea }\end{array}$ \\
\hline 4 & $\begin{array}{l}\text { Multíparas sin cesárea previa, con un feto en presentación cefálica, de } 37 \\
\text { semanas o más de embarazo, que han sido sometidas a inducción o a cesárea } \\
\text { antes del inicio del parto }\end{array}$ \\
\hline $5 \mathrm{~A}$ & $\begin{array}{l}\text { Multíparas con una cesárea previa, con un feto único en presentación cefálica, } \\
\text { de } 37 \text { semanas o más de embarazo }\end{array}$ \\
\hline 5B & $\begin{array}{l}\text { Multíparas con más de una cesárea previa, con un feto único en presentación } \\
\text { cefálica, de } 37 \text { semanas o más de embarazo }\end{array}$ \\
\hline 6 & Nuliparas con un feto único en presentación podálica \\
\hline 7 & $\begin{array}{l}\text { Multiparas con un feto único en presentación podálica, incluyendo aquéllas } \\
\text { con cesárea previa }\end{array}$ \\
\hline 8 & $\begin{array}{l}\text { Todas las mujeres con embarazo múltiple, incluyendo aquéllas con cesárea } \\
\text { previa }\end{array}$ \\
\hline 9 & $\begin{array}{l}\text { Todas las mujeres con feto único en posición transversa u oblicua, incluyendo } \\
\text { aquéllas con cesárea previa }\end{array}$ \\
\hline 10 & $\begin{array}{l}\text { Todas las mujeres con un feto único en presentación cefálica de menos de } 37 \\
\text { semanas de embarazo, incluyendo aquéllas con cesárea previa }\end{array}$ \\
\hline
\end{tabular}

Fuente: elaboración propia

\section{OBJETIVOS}

El objetivo de este estudio es analizar la tasa de cesáreas anual y en los 10 años y 10 meses en las dos maternidades públicas de referencia de Uruguay: Hospital de Clinicas ( $\mathrm{HC}$ ) y Centro Hospitalario Pereira Rossell (CHPR) y compararlos entre si identificando los grupos de Robson con mayor incidencia de las mismas.

\section{NORMAS ÉTICAS}

El presente estudio es retrospectivo a partir de datos obtenidos del Sistema Informático Perinatal (SIP), se preservaron la confidencialidad, anonimato y derechos de las mujeres participantes, considerándose una investigación sin riesgos ya que el sujeto de investigación no sufre daño alguno como consecuencia inmediata o tardia del estudio.

El estudio cuenta con el aval y aprobación del Comité de Ética en Investigación del Centro Hospitalario Pereira Rossell (comité.etica.investigacion@gmail.com).

\section{MATERIAL Y MÉTODOS}

Se realizó un estudio observacional, descriptivo, retrospectivo y transversal para analizar la vía de finalización de los nacimientos, así como las caracteristicas demográficas de las poblaciones en las maternidades del HC y CHPR en un periodo de 10 años y 10 meses desde el 1/1/2009 al 31/10/2019. Los datos se obtuvieron a partir del SIP, este es un sistema publicado por el Centro Latinoamericano de Perinatología, Salud de la Mujer y Reproductiva en 1983. utilizado como estrategia para mejorar la calidad de la atención a madres y recién nacidos ${ }^{(7)}$. Actualmente el Ministerio de Salud Pública de Uruguay exige su uso por parte de todas las maternidades del país, y se encarga de recolectar y validar los datos ingresados con el objetivo de obtener estadisticas fiables a nivel institucional, local y nacional. El mismo contiene información demográfica, antecedentes obstétricos, atención prenatal, finalización del embarazo, complicaciones, parto y puerperio y desenlaces neonatales, datos recopilados por médicos y obstetras, prospectivamente, a partir del primer control prenatal hasta el alta de la madre y el recién nacido(7).

Tanto el HC como el CHPR, son centros de atención de tercer nivel que reciben pacientes obstétricas provenientes tanto de Montevideo como del interior del país que se asisten en el sistema de salud pública del Uruguay. Sin embargo, si bien ambos cuentan con Centro de Tratamiento Intensivo (CTI) neonatal para la atención de neonatos de bajo, mediano y alto riesgo, únicamente el HC cuenta con CTI materno y siendo un hospital que nuclea todas las especialidades médicas y quirúrgicas, cuenta con centro de diálisis, con centro de cirugía cardiaca, piso de neurocirugía entre otras tantas especialidades lo que lo hace recibir pacientes obstétricas de alta complejidad en su manejo.

En este estudio se incluyeron todas las mujeres que tuvieron sus partos por vía vaginal o cesárea en estos dos centros en el periodo de tiempo mencionado. Se excluyeron a las pacientes con embarazos menores de 20 semanas.

Se utilizó la clasificación de diez grupos propuesta por Robson para cada centro. Se obtuvo la información necesaria de cada mujer embarazada para esta clasificación:

1) embarazo con feto único/múltiple;

2) paridad;

3) inicio del parto;

4) edad gestacional;

5) cesárea previa; y

6) presentación o situación fetal.

Se consideró nuliparas a las pacientes sin antecedentes de partos o cesáreas previas y multiparas 
a las pacientes con al menos un parto o cesárea previa. Se calificó el inicio de parto espontáneo cuando no hubo intervención médica para desencadenar el inicio de las contracciones uterinas; inducido cuando se dio una intervención farmacológica o mecánica para hacerlo; y cesárea electiva cuando se programó la cesárea antes de que ocurriera alguno de estos dos eventos. La edad gestacional se definió por ecografia. El antecedente de cicatriz uterina incluyó la presencia de una o más cesáreas. La presentación o situación fetal se categorizó en cefálica, podálica, transversa u oblicua. La tasa de cesárea se calculó como la proporción de pacientes que tuvieron parto por cesárea del total de los partos atendidos en el lapso de tiempo mencionado, calculado para cada centro, en cada año y de forma global.

Las pacientes se clasificaron de acuerdo a los diez grupos clínicos, mutuamente excluyentes, descriptos por Robson. Se calculó, para cada centro por separado:

1. El tamaño de cada grupo como la relación entre las pacientes pertenecientes a cada grupo de Robson con respecto al total de la población atendida ([número de pacientes atendidas pertenecientes a cada grupo/ número total de nacimientos] $\times 100$ ).

2. La proporción especifica de cesáreas realizada dentro de cada grupo (Inúmero de cesáreas realizadas a mujeres pertenecientes a cada grupo/ número de nacimientos pertenecientes a ese mismo grupo] x100).

3. La contribución absoluta hecha por cada grupo al porcentaje global de cesáreas como el dividendo del número de cesáreas en el grupo por el total de partos atendidos (Inúmero total de cesáreas realizadas en el grupo /número total de nacimientos] x100).

4. La contribución relativa del grupo al porcentaje global de cesáreas como el dividendo del número de cesáreas del grupo por el número total de cesáreas de cada centro (Inúmero de cesáreas en el grupo/número total de cesáreas en cada centro] x100).

Para comparar ambos centros se utilizó el test de chi cuadrado y se consideró estadisticamente significativo un Valor p menor a 0,05 utilizando el programa OpenEpi versión 3.01 con tabla de 2x2 disponible en: http:// www.openepi.com/TwobyTwo/TwobyTwo.htm.

\section{RESULTADOS}

Se obtuvo un total de 85.526 nacimientos. De estos nacimientos $7.685(8,9 \%)$ se produjeron en el HC mientras que 77.841 (91.1\%) fueron en el CHPR.

En la Tabla 2 se muestran las características de la población para cada centro. En el HC 3.006 mujeres (39,1\%) eran nuliparas y $4.676(60,9 \%)$ multiparas, versus $26.067(33.5 \%)$ y $51.774(66.5 \%)$ en el CHPR respectivamente. En el HC 1.632 (21,2\%) tenían el antecedente obstétrico de una o más cesáreas mientras que en el CHPR fue el 12.621 (16,2\%).

Respecto a la forma de inicio del parto en el HC 3.997 (52\%) fueron de inicio espontaneo, 1.847 (24\%) fueron de inicio inducido y 1.841 (24\%) fueron cesáreas electivas; en el CHPR 50.873 (65.3\%), $15.735(20,3 \%$ ) y $11.233(14,4 \%)$ respectivamente.

Respecto al porcentaje de cesáreas en cada centro y por año se observa un porcentaje de cesáreas por año de $49,2 \% \pm 5$ en el HC vs $29,3 \% \pm 3$ en CHPR.

Tabla 2. Datos previos al embarazo y del embarazo

\begin{tabular}{|c|c|c|c|c|c|}
\hline \multirow{2}{*}{ Caracteristica } & Categoria & \multicolumn{2}{|c|}{$\begin{array}{c}\text { Hospital de } \\
\text { Clinicas }\end{array}$} & \multicolumn{2}{c|}{$\begin{array}{c}\text { Centro Hospitalario } \\
\text { Pereira Rossell }\end{array}$} \\
\cline { 3 - 6 } & & $\mathrm{N}$ & $\%$ & $\mathrm{n}$ & $\%$ \\
\hline \multirow{2}{*}{ Paridad } & Nulipara & 3.006 & $39.1 \%$ & 26.067 & $33.5 \%$ \\
\cline { 2 - 6 } & Multípara & 4.679 & $60.9 \%$ & 51.774 & $66.5 \%$ \\
\hline \multirow{2}{*}{$\begin{array}{c}\text { Antecedente de 10 } \\
\text { más cesáreas }\end{array}$} & $\mathrm{Si}$ & 1.632 & $21.2 \%$ & 12.621 & $16.2 \%$ \\
\hline \multirow{2}{*}{$\begin{array}{c}\text { Forma de inicio del } \\
\text { parto }\end{array}$} & Espontáneo & 3.053 & $78,8 \%$ & 65.220 & $83.8 \%$ \\
\cline { 2 - 6 } & Inducido & 1.847 & $24 \%$ & 15.735 & $20.3 \%$ \\
\cline { 2 - 6 } & Cesárea electiva & 1.841 & $24 \%$ & 11.233 & $14.4 \%$ \\
\hline $\begin{array}{c}\text { Vía de finalización del } \\
\text { embarazo }\end{array}$ & $\begin{array}{c}\text { Parto vaginal } \\
\text { normal }\end{array}$ & 3.468 & $45.2 \%$ & 54.188 & $69.9 \%$ \\
\cline { 2 - 6 } & Cesárea & 3.849 & $50 \%$ & 21.095 & $27.1 \%$ \\
\cline { 2 - 6 } & $\begin{array}{c}\text { Parto } \\
\text { instrumental }\end{array}$ & 368 & $4,8 \%$ & 2.558 & $3.3 \%$ \\
\hline
\end{tabular}

Características demográficas en cuanto paridad, antecedente de 1 o más cesáreas, forma de inicio del trabajo de parto y vía de finalización del embarazo.

Fuente: elaboración propia.

En la Figura 1 se puede valorar la evolución del porcentaje de cesáreas en cada año para cada centro. Para el HC se observa desde 2009 al 2019 los siguientes porcentajes de cesárea por año: 32\%, 37,2\%, $44,2 \%, 43,2 \%, 49 \%, 46,4 \% 46,5 \%, 47,6 \%, 46,5 \%, 51,3 \%$ y $47,4 \%$ respectivamente. Mientras que los porcentajes de cesáreas por año para el CHPR fueron: $23,1 \%, 24,4 \%$, $26,9 \%, 26,3 \%, 28,7 \%, 27,1 \%, 25,3 \% 24,4 \%, 27,9 \%, 29 \%$ y $30,3 \%$ respectivamente.

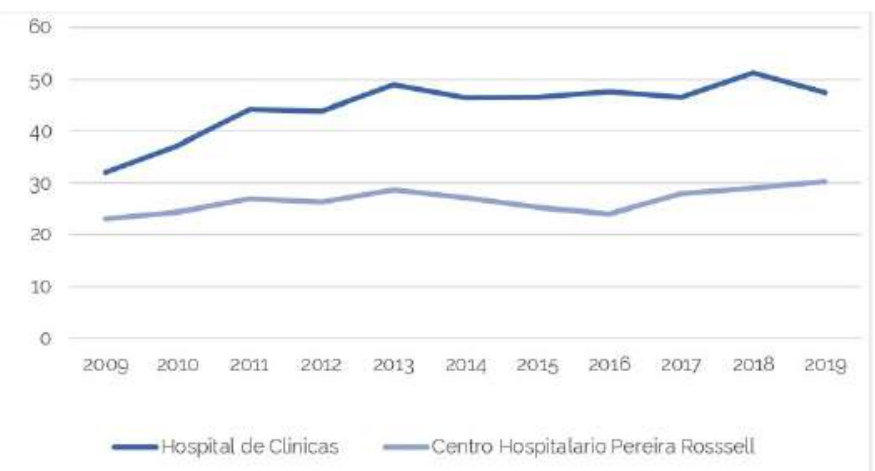

Figura 1. Porcentaje de cesáreas por año para cada centro (\%) Fuente: elaboración propia.

En la Tabla 3 y $\mathbf{4}$ se muestran la distribución de pacientes utilizando la clasificación de Robson el HC y CHPR respectivamente, mientras que en la Figura 2 permite comparar dicha distribución entre los dos centros.

En el $\mathrm{HC}$ los grupos 1,5A y 10 registraron mayor 
número de pacientes con 21,4\%, 16,1\% y $14,7 \%$ respectivamente. Por otro lado, los grupos que registraron mayor número de pacientes en el $\mathrm{CHPR}$ fueron el 3, 1 y 5A, en ese orden con $32,4 \%, 20,5 \%$ y 13,1\% respectivamente.

Tabla 3. Clasificación de Robson Hospital de Clínicas

\begin{tabular}{|c|c|c|c|c|c|}
\hline $\begin{array}{c}\text { Categorias } \\
\text { de Robson }\end{array}$ & $\begin{array}{c}\text { Cesárea } \\
\text { /parto } \\
\text { vaginal }\end{array}$ & $\begin{array}{c}\text { Tamaño } \\
\text { del grupo } \\
\text { (\%) }\end{array}$ & $\begin{array}{c}\% \\
\text { Cesárea }\end{array}$ & $\begin{array}{c}\text { Contribución } \\
\text { absoluta del } \\
\text { grupo a la tasa } \\
\text { global de } \\
\text { cesáreas (\%) } \\
\text { n cesáreas del } \\
\text { grupo / N } \\
\text { total de } \\
\text { mujeres que } \\
\text { dan a luz en el } \\
\text { hospital } \times 100\end{array}$ & $\begin{array}{c}\text { Contribución } \\
\text { relativa del } \\
\text { grupo a la tasa } \\
\text { global de } \\
\text { cesáreas (\%) } \\
\text { n cesáreas del } \\
\text { grupo / N total } \\
\text { cesáreas del } \\
\text { hospital x100 }\end{array}$ \\
\hline 1 & $460 / 1184$ & $21,4 \%$ & $28 \%$ & $5.9 \%$ & $11,9 \%$ \\
\hline 2 & $431 / 583$ & $13,2 \%$ & $42,6 \%$ & $5,6 \%$ & $11,2 \%$ \\
\hline 3 & $188 / 327$ & $6,7 \%$ & $36,6 \%$ & $2,4 \%$ & $4.9 \%$ \\
\hline 4 & $193 / 821$ & $13,2 \%$ & $19,1 \%$ & $2.5 \%$ & $5 \%$ \\
\hline $5 \mathrm{~A}$ & $798 / 439$ & $16,1 \%$ & $64,5 \%$ & $10,3 \%$ & $20,7 \%$ \\
\hline $5 \mathrm{~B}$ & $363 / 6$ & $4.8 \%$ & $98.4 \%$ & $4.7 \%$ & $9.4 \%$ \\
\hline 6 & $99 / 9$ & $1,4 \%$ & $92 \%$ & $1,3 \%$ & $2,6 \%$ \\
\hline 7 & $192 / 16$ & $2,7 \%$ & $92,4 \%$ & $2,5 \%$ & $5 \%$ \\
\hline 8 & $353 / 16$ & $4,8 \%$ & $95,7 \%$ & $4,6 \%$ & $9,2 \%$ \\
\hline 9 & $75 / 2$ & $1 \%$ & $98,1 \%$ & $0,9 \%$ & $1,8 \%$ \\
\hline 10 & $688 / 442$ & $14.7 \%$ & $60,9 \%$ & $8,9 \%$ & $17.8 \%$ \\
\hline
\end{tabular}

Se muestra tamaño de cada grupo, \% de cesáreas y contribución absoluta y relativa a la tasa global de cesáreas en el HC

Fuente: elaboración propia.

Tabla 4. Clasificación de Robson Centro Hospitalario Pereira Rossell

\begin{tabular}{|c|c|c|c|c|c|}
\hline $\begin{array}{l}\text { Categorias } \\
\text { de Robson }\end{array}$ & $\begin{array}{l}\text { Cesárea } \\
\text { /Parto } \\
\text { vaginal }\end{array}$ & $\begin{array}{c}\text { Tamaño } \\
\text { del grupo } \\
(\%)\end{array}$ & $\begin{array}{c}\% \\
\text { Cesárea }\end{array}$ & $\begin{array}{c}\text { Contribución } \\
\text { absoluta del } \\
\text { grupo a la tasa } \\
\text { global de } \\
\text { cesáreas (\%) } \\
\text { n cesáreas del } \\
\text { grupo / N total } \\
\text { de mujeres } \\
\text { que dan a luz } \\
\text { en el hospital x } \\
100\end{array}$ & $\begin{array}{c}\text { Contribución } \\
\text { relativa del } \\
\text { grupo a la tasa } \\
\text { global de } \\
\text { cesáreas (\%) } \\
\text { n cesáreas del } \\
\text { grupo / N total } \\
\text { cesáreas del } \\
\text { hospital x100 }\end{array}$ \\
\hline 1 & $\begin{array}{c}2.724 / 13 \\
309\end{array}$ & $20,6 \%$ & $17 \%$ & $3.5 \%$ & $12.9 \%$ \\
\hline 2 & $\begin{array}{c}2.136 / 3.7 \\
80\end{array}$ & $7,6 \%$ & $36,1 \%$ & $2,7 \%$ & $10,1 \%$ \\
\hline 3 & $\begin{array}{c}984 / 24.2 \\
36\end{array}$ & $32,4 \%$ & $3.9 \%$ & $1,3 \%$ & $4,6 \%$ \\
\hline 4 & $\begin{array}{c}922 / 2.50 \\
5\end{array}$ & $4.4 \%$ & $26,9 \%$ & $1,2 \%$ & $4,4 \%$ \\
\hline $5^{a}$ & $\begin{array}{c}4.562 / 5.7 \\
12\end{array}$ & $13,2 \%$ & $44,4 \%$ & $5.9 \%$ & $21,6 \%$ \\
\hline $5 \mathrm{~B}$ & $2.276 / 60$ & $3 \%$ & $97.4 \%$ & $2,9 \%$ & $10,7 \%$ \\
\hline 6 & $1.027 / 142$ & $1,5 \%$ & $87,8 \%$ & $1,3 \%$ & $4.9 \%$ \\
\hline 7 & $\begin{array}{c}1.301 / 25 \\
8\end{array}$ & $2 \%$ & $83.5 \%$ & $1.7 \%$ & $6,2 \%$ \\
\hline 8 & $\begin{array}{c}2.132 / 36 \\
0\end{array}$ & $3.2 \%$ & $85.6 \%$ & $2.7 \%$ & $10.1 \%$ \\
\hline 9 & $370 / 21$ & $0.5 \%$ & $94,6 \%$ & $0.5 \%$ & $1,7 \%$ \\
\hline 10 & $2.661 / 6.3$ & $11,6 \%$ & $29.5 \%$ & $3.4 \%$ & $12,6 \%$ \\
\hline
\end{tabular}

Se muestra tamaño de cada grupo, \% de cesáreas y contribución absoluta y relativa a la tasa global de cesáreas en el CHPR

Fuente: elaboración propia.

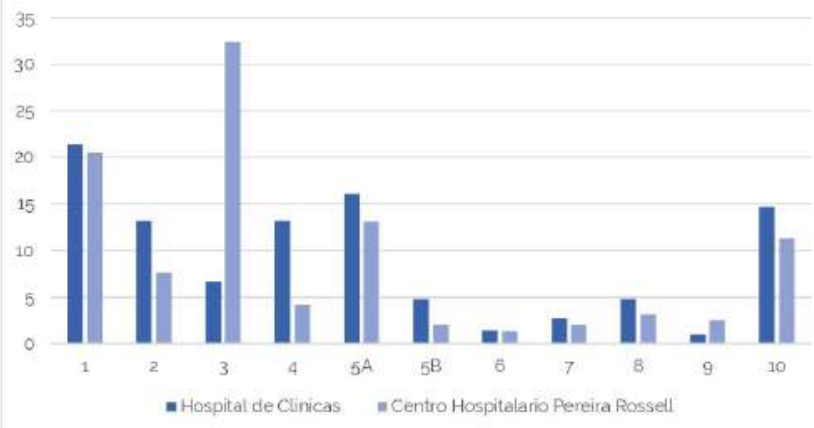

Figura 2. Porcentaje para cada categoria de Robson en ambos centros (\%) Fuente: elaboración propia.
En la Tabla 5 se compara el porcentaje de pacientes para cada grupo en cada centro, observándose que únicamente los grupos 1 y 6 no tienen diferencias estadisticamente significativas (Valor p mayor a 0,05), mientras que el resto de los grupos tienen diferencias significativas.

Tabla 5. Porcentaje de pacientes para cada categoría de Robson en ambos centros

\begin{tabular}{|c|c|c|c|c|c|}
\hline \multirow[t]{2}{*}{$\begin{array}{l}\text { Categorias } \\
\text { de Robson }\end{array}$} & \multicolumn{2}{|c|}{ Hospital de Clínicas } & \multicolumn{2}{|c|}{$\begin{array}{c}\text { Centro Hospitalario } \\
\text { Pereira Rossell }\end{array}$} & \multirow[t]{2}{*}{ Valor $\mathrm{p}$} \\
\hline & $n$ & $\%$ & $\mathrm{n}$ & $\%$ & \\
\hline 1 & 1.644 & $21.4 \%$ & 16.033 & $20,6 \%$ & 0,1005 \\
\hline 2 & 1.014 & $13.2 \%$ & 5.916 & $7,6 \%$ & $<0,001$ \\
\hline 3 & 515 & $6,7 \%$ & 25.220 & $32,4 \%$ & $<0,001$ \\
\hline 4 & 1.014 & $13,2 \%$ & 3.427 & $4.4 \%$ & $<0,001$ \\
\hline $5 \mathrm{~A}$ & 1.237 & $16,1 \%$ & 10.274 & $13,2 \%$ & $<0,001$ \\
\hline $5 \mathrm{~B}$ & 369 & $4,8 \%$ & 2.336 & $3 \%$ & $<0,001$ \\
\hline 6 & 108 & $1,4 \%$ & 1.169 & $1,5 \%$ & 0,5060 \\
\hline 7 & 208 & $2,7 \%$ & 1.559 & $2 \%$ & $<0,001$ \\
\hline 8 & 369 & $4,8 \%$ & 2.492 & $3,2 \%$ & $<0,001$ \\
\hline 9 & 77 & $1 \%$ & 391 & $0,5 \%$ & $<0,001$ \\
\hline 10 & 1.130 & $14.7 \%$ & 9.024 & $11,6 \%$ & $<0,001$ \\
\hline
\end{tabular}

Fuente: elaboración propia.

Los grupos con mayor contribución relativa a la tasa global de cesáreas en el HC fueron los: $5 \mathrm{~A}, 10 \mathrm{y}$ 1 con tasas de $20,8 \%, 17,4 \%$ y $12,1 \%$ respectivamente. Los grupos que tuvieron mayor contribución en el CHPR fueron los mismos con 21,8\%, 12,5\% y 12,9\% respectivamente. La contribución relativa del grupo a la tasa global de cesáreas para cada centro se puede ver en la Figura 3. En la Tabla 6 se comparan dichas tasas para cada grupo y cada centro destacando que no existen diferencias estadisticamente significativas en la mayoria de las categorias para ambos centros a excepción de las categorias 2, 5B, 6, 7 y 10.

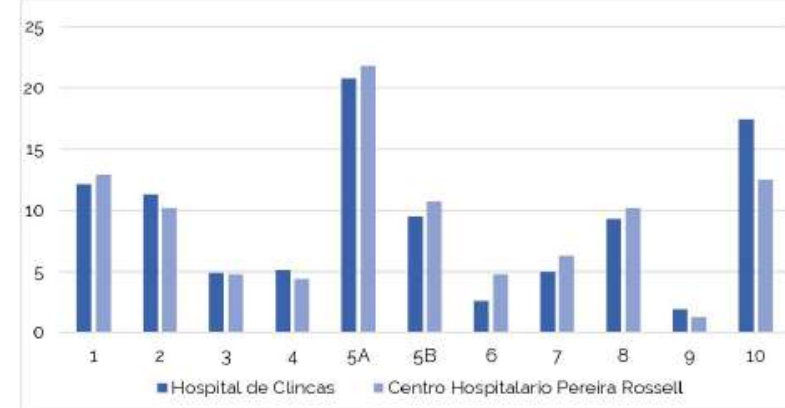

Figura 3. Contribución relativa del grupo a la tasa global de cesáreas para cada centro (\%)

Fuente: elaboración propia.

Tabla 6. Contribución relativa del grupo a la tasa global de cesáreas para cada centro.

\begin{tabular}{|c|c|c|c|c|c|}
\hline \multirow[t]{2}{*}{$\begin{array}{l}\text { Categorias } \\
\text { de Robson }\end{array}$} & \multicolumn{2}{|c|}{ Hospital de Clínicas } & \multicolumn{2}{|c|}{$\begin{array}{l}\text { Centro Hospitalario } \\
\text { Pereira Rossell }\end{array}$} & \multirow[t]{2}{*}{ Valor $\mathrm{p}$} \\
\hline & $\mathrm{n}$ & $\%$ & $\mathrm{n}$ & $\%$ & \\
\hline 1 & 460 & $11.9 \%$ & 2.724 & $12,9 \%$ & 0,1001 \\
\hline 2 & 431 & $11,2 \%$ & 2.136 & $10,1 \%$ & 0,0441 \\
\hline 3 & 188 & $4.9 \%$ & 984 & $4.6 \%$ & 0.5535 \\
\hline 4 & 193 & $5 \%$ & 922 & $4,4 \%$ & 0,0756 \\
\hline $5^{a}$ & 798 & $20.7 \%$ & 4.562 & $21,6 \%$ & 0,2151 \\
\hline $5 \mathrm{~B}$ & 363 & $9.4 \%$ & 2.276 & $10.7 \%$ & 0,0117 \\
\hline 6 & 99 & $2,6 \%$ & 1.027 & $4.9 \%$ & $<0,001$ \\
\hline 7 & 192 & $5 \%$ & 1.301 & $6,2 \%$ & 0,0045 \\
\hline 8 & 353 & $9.2 \%$ & 2.132 & $10,1 \%$ & 0,0747 \\
\hline 9 & 75 & $1,8 \%$ & 370 & $1,7 \%$ & 0,4016 \\
\hline 10 & 688 & $17.8 \%$ & 2.661 & $12,6 \%$ & $<0,001$ \\
\hline
\end{tabular}

Fuente: elaboración propia. 


\section{DISCUSIÓN}

En 2015 la OMS realizó una declaración en donde recomendaba que la tasa ideal de cesárea debe oscilar entre el $10 \%$ y el $15 \%$ (3). Para ello se basó en la declaración hecha por un panel de expertos en salud reproductiva en una reunión organizada por la OMS en 1985 en Fortaleza (Brasil)(8). Las cesáreas son cada vez más frecuentes, tanto en países desarrollados como en paises en desarrollo(2,9). En Latinoamérica nacen cerca de 11 millones de niños cada año y las tasas de cesárea alcanzan valores que superan el $35 \%$, lo que representa 2 millones de cesáreas adicionales por año(10). Uruguay no resulta ajeno a esta tendencia, pues su tasa de cesáreas pasó de un 32\% en 2006 a un 43,1\% en 2015 $5^{(11)}$. El aumento en la tasa de cesáreas asocia riesgos evidenciado un aumento de las complicaciones siendo las más frecuentes: infecciones, hemorragia obstétrica, histerectomía periparto, necesidad de unidad de cuidados intensivos y acretismo placentario ${ }^{(12)}$.

En nuestro estudio podemos evidenciar que en ambos centros ha habido un aumento en la tasa global de cesáreas entre el 2009 al 2019, pasando del $32 \%$ a $47,4 \%$ en el $\mathrm{HC}$ y del $23,1 \%$ al $30,3 \%$ en el CHPR, lo cual no escapa a las estadísticas internacionales y nacionales mencionadas anteriormente. Existen múltiples razones que pueden explicar el aumento en la tasa de cesáreas siendo las 4 más referidas: un número mayor de usuarias que solicitan cesáreas a demanda o sin justificación médica, aumento de los litigios médico-legales que llevan a un mayor intervencionismo obstétrico, aumento en el número de mujeres que realmente tienen indicación médica de cesárea y un beneficio de tiempos y económico para el médico tratante ${ }^{(13)}$.

En relación a los grupos de Robson más prevalentes en ambos centros, mientras que para el $\mathrm{HC}$ fueron los grupos 5A, 1 y 10 para el CHPR fueron los grupos 1 , 3 y $5 \mathrm{~A}$. De estos datos podemos analizar que, si bien los grupos 1 y $5 \mathrm{~A}$ son los más prevalentes en ambos centros, el HC presenta mayor porcentaje respecto al CHPR del grupo 5A, con diferencia estadísticamente significativas, el cual podria asociarse a mayor riesgo de cesárea. Por otro lado, el CHPR tiene un alto porcentaje de pacientes pertenecientes al grupo 3 las cuales son las que presentan menor riesgo de cesárea. Mientras que el $\mathrm{HC}$ presenta mayor porcentaje de embarazos de pretérmino, con mayor riesgo de cesárea, existiendo diferencias estadisticamente significativas en los grupos mencionadas para los dos centros.

Por otro lado, los grupos de Robson con mayor contribución relativa a la tasa global de cesáreas son el 1, 5A y 10 para ambos centros, sin diferencias estadisticamente significativas para los grupos 1 y $5 \mathrm{~A}$ entre los centros. Existen múltiples recomendaciones de disminuir la realización de la primera cesárea en embarazos de bajo riesgo, es decir, embarazos simples, con fetos únicos en presentación cefálica y de termino, como es el caso del grupo 1 de la clasificación de Robson $^{(14,15)}$. El aumento en la tasa de cesáreas de este grupo determina un aumento en la tasa de cesáreas en las pacientes multiparas al aumentar el factor de cesárea previa, como es el grupo 5A de la clasificación de Robson ${ }^{(14)}$. El Colegio Americano de Ginecología y Obstétrica publicó en 2014 una recomendación acerca de la prevención de la primera cesárea teniendo en cuenta múltiples variables, entre ellas, la progresión del trabajo de parto la cual sería considerablemente mayor en pacientes nuliparas y por tanto sobrepasar estos plazos no debería ser una indicación estricta de cesárea ${ }^{(15)}$

La via de finalización del embarazo en el grupo de pacientes con cesáreas previas ha sido, por mucho tiempo, controversial lo que podría determinar un grupo con un mayor riesgo de cesárea. Sin embargo, el Colegio Americano de Ginecología y Obstetricia recomienda fomentar la prueba de parto vaginal en gestantes con cesárea anterior sin contraindicaciones para la misma, las cuales tienen un éxito de parto vaginal de entre el 60 al $80 \%$ y con pocas complicaciones ${ }^{(16)}$. En cuanto a los beneficios de un parto vaginal post cesárea si bien la mortalidad materna es mayor que en una cesárea electiva, su valor relativo sigue siendo muy bajo (13/100.000 vs 4/100.000) por lo que el parto post cesárea es una práctica segura, considerando siempre los deseos de cada paciente ${ }^{(17)}$. En relación a la morbilidad el riesgo de rotura uterina en el parto post cesárea, la cual es la complicación más temida, se equipara con el mayor riesgo de histerectomía en futuros embarazos vinculados a trastornos adherenciales, placentarios y complicaciones quirúrgicas ${ }^{(17)}$.

Con respecto a la contribución relativa a la tasa de cesárea de los otros grupos de Robson tampoco existen diferencias estadisticamente significativas para los grupos 2, 3, 4, 8 y 9 entre ambos centros. Sin embargo, existen diferencias significativas para los grupos 5B, 6, 7 y 10 lo que podría explicarse por las diferentes políticas de asistencia de los centros en cuanto al parto en presentaciones distocias. A su vez, el HC se caracteriza por ser un centro que asiste embarazos con patológicas médicas y obstétricas de alto riesgo como son: preeclampsias severas que requieran ingreso a CTI materno, nefropatías, cardiopatias y enfermedades autoinmunes, entre otras. Esto último podria estar relacionado a la necesidad de interrupción de los embarazos a edades gestacionales precoces que, en el CHPR, lo que podría explicar la mayor tasa de cesáreas en este grupo.

Encontramos como limitantes del estudio no 
lograr identificar otras patologías graves que no son consideradas en la clasificación de Robson y que puedan preponderar en las poblaciones que estudiamos. La posibilidad de que aumenten el número de cesáreas, merece mayor estudio.

\section{CONCLUSIONES}

Este estudio pone en evidencia que ninguna de las dos maternidades públicas de referencia del Uruguay escapa a las estadisticas internacionales en cuanto al aumento en la tasa de cesáreas. Dado que cada centro posee poblaciones diferentes, la clasificación de Robson permite valorar y comparar la tasa de cesáreas según subgrupos, evidenciado que, si bien la tasa global de cesáreas del HC es mayor que en el CHPR al analizar los grupos por separado, la tasa de cesáreas es similar en ambos centros. Esta discordancia podria explicarse por las características poblacionales de cada maternidad, destacando que mientras que el CHPR se caracteriza por la atención de pacientes de bajo riesgo obstétrico, el $\mathrm{HC}$ recibe mayor porcentaje de pacientes de alto riesgo obstétrico siendo centro de referencia nacional por contar con CTI materno y una amplia gama de especialidades médico-quirúrgicas.

Finalmente, es importante destacar la importancia de reducir el porcentaje de la primera cesárea en ambas maternidades. A su vez, en ambos centros se debería fomentar el parto vaginal en mujeres con cesáreas previas reforzando protocolos ya establecidos.

\section{Agradecimientos:}

Se agradece a los profesores titulares de cada clínica ginecotocológica: Dr. Washington Lauria (Clinica Ginecotocológica 'B', Hospital de Clinicas Dr. Manuel Quintela) y Dr. Claudio Sosa (Clínica Ginecotocológica 'C', Hospital Pereira Rossell).

\section{REFERENCIAS}

1. Betrán AP, Ye J, Moller AB, Zhang J, Gulmezoglu AM, Torloni. The increasing trend in caesarean section rates: Global,regional and national estimates: 19902014. PLOS ONE. 2016. 11(2): e0148343

2. Vogel JP, Betrán AP, Vindevoghel N, Souza JP, Torloni MR, Zhang $J$ et al. Use of the Robson classification to assess caesarean section trends in 21 countries: a secondary analysis of two WHO multicountry surveys on behalf of the WHO MultiCountry Survey on Maternal and Newborn Health Research Network. Lancet Global Health. 2015. 3(5):e260-70.

3. Organización Mundial de la Salud, HRP. Declaración de la OMS sobre tasas de cesárea.. 2015. WHO/RHR/15.02. disponible en: https://apps.who.int/iris/bitstream/ handle/10665/161444/WHO RHF 15.02 spa. pdf:jsessionid=C102AC4D93CD5EE78A976EB1D08E7E?sequ

4. Betrán AP, et al. What is the optimal rate of caesarean section a population level? A systematic review of ecologic studies. Reprodcutive Health. 2015. 12(57)

5. Souza JP, Gülmezoglu AM, P Lumbiganon, M Laopaiboon, G Carroli, B Fawoleet al. Caesarean section without medical indications is associated with an increased risk of adverse shortterm maternal outcomes: the 20042008 WHO Global Survey on Maternal and Perinatal Health. BMC medicine. 2010. 8(71).

6. Organización Panamericana de la Salud. La clasificación de Robson: Manual de aplicación.. 2018.

7. Organización Panamericana de la Salud. Sistema Informático Perinatal... [En linea]. Disponible en: https://wwww.paho. org/clap/index.php?option=com_content\&view=article\&i $\underline{d=84}$ : sistema-informatico-perinatal\&ltemid=242\&lang $=e n$ [Consultado: 15 Abr 2020].

8. Organización Mundial de la Salud. Appropriate technology for birth. Lancet. 1985. 2(8452):436-7.

9. Ye J, Betran AP, Vela MG, Souza JP, Zhang J. Searchng for the Optimal Rate of Medically Necessary Cesarean Delivery. Birth. 2014. 41(3):237-43.

10. Ruiz-Sánchez J, Espino y Sosa S, Vallejos-Parés A,§ DuránArenas L. Cesarea Tendencias y resultados. Perinatología y reproduccion humana. 2014. 28(1):33-40.

11. Dirección de Descentralización e Inversión Pública. OPP Observatorio Territorio Uruguay. Índice de cesáreas. Ien linea]. Disponible en: https://otu.opp.gub.uy/[Consultado 22 Abr 2020].

12. Villar J, Valladares E, Wojdyla D, Zavaleta N, Carroli G, Velazco A, et al. Caesarean delivery rates and pregnancy outcomes: the 2005 WHO global survey on maternal and perinatal health in Latin America. Lancet. 2006. 367(9525):1819-26.

13. Cóppola F. Cesáreas en Uruguay. Rev. Méd. Urug. 2015 31(1):7-14. [en linea]. Disponible en: http://www.scielo. edu.uy/scielo.php?script=sci_arttext\&pid=S1688$03902015000100002 \&$ lng $=e s$. [Consultado 20 Apr 2020].

14. Brennan D, Murphy M, Robson MS, O'Herlihy C. The singleton, cephalic, nulliparous woman after 36 weeks of gestation: contribution to overall cesarean delivery rates. Obstet Gynecol. 2011. 117:273-9.

15. Caughey AB, Cahill AG, Guise JM, Rouse DJ. Safe prevention of the primary cesarean delivery. Obstetrical and Gynecological Survey. 2014.69(7):981-383.

16. American College of Obstetricians and Gynecologists Vaginal Birth After Cesarean (ACOG Practice Bulletin No. 5) International Journal of Gynecology and Obstetricis. 1999. 66(2):197-204

17. Sociedad de obstetricia y ginecologia de Buenos Aires. Consenso. Parto vaginal despues de una cesarea. 2019.

\section{Nota del autor:}

Los autores declaran no tener conflicto de intereses. El estudio no recibió financiación de ningún tipo.

\section{Nota de contribución:}

Dra. Florencia Feldman: Concepción y diseño del estudio, Análisis e interpretación de los datos o resultados, Análisis estadísticos de los datos o resultados.

Dra. Gina Del Villar: Recolección de los datos, Elaboración de un borrador del manuscrito, Concepción y diseño del estudio, Análisis e interpretación de los datos o resultados.

Dra Pamela Grimaldi: Elaboración de un borrador del manuscrito. Dr. Santiago Scasso: Supervisión del trabajo.

Dra. Grazzia Rey: Supervisión del trabajo.

\section{Nota del Editor:}

El presente manuscrito fue aprobado por Enrique Pons como su editor.

Recibido: 14/02/2021

Aceptado: 02/03/2021 